\title{
Olfactory functioning in early multiple sclerosis: Sniffin' Sticks Test study
}

This article was published in the following Dove Press journal:

Neuropsychiatric Disease and Treatment

26 August 2016

Number of times this article has been viewed

\author{
Hale Z Batur Caglayan' \\ Ceyla Irkec' \\ Bijen Nazliel' \\ Aslı Akyol Gurses ${ }^{2}$ \\ Irem Capraz'
}

'Department of Neurology, Faculty of Medicine, Gazi University, Ankara, ${ }^{2}$ Department of Neurology, Meram Faculty of Medicine, Necmettin Erbakan University, Konya, Turkey
Correspondence: Hale Z Batur Caglayan Department of Neurology, Faculty of Medicine, Gazi University, C-Block 3rd Floor, Besevler, 06500, Cankaya/Ankara, Turkey

Tel +90312 2025344

Email halezeynep@gazi.edu.tr
Introduction: Previous studies have shown that olfactory functioning is affected by multiple sclerosis (MS). This study assessed the level of the olfactory impairment in early MS by using the Sniffin' Sticks Test.

Methods: This study included 30 patients with MS and 30 healthy controls. We collected demographic and clinical data from participants and administered the Sniffin' Sticks Test.

Results: We found no differences between the MS and control groups in odor discrimination, odor identification, and threshold discrimination identification scores, but odor threshold (OT) scores were higher in the control group than in the MS group $(P=0.49)$. In addition, we did not find any correlation between MS patients' olfactory test scores and their scores on the Mini-Mental State Examination (MMSE), Expanded Disability Status Scale (EDSS), disease duration, history of optic neuritis, or being on immunomodulatory therapy.

Conclusion: In recent studies, odor threshold impairment seemed to be the most striking finding in patients with MS. Although the present study found a mild alteration in odor threshold, olfactory dysfunction appears to be a consequence of neurodegeneration in the higher order olfactory brain regions, which is thought to be a time-dependent process.

Keywords: demyelinating diseases, multiple sclerosis, odors, olfaction, olfaction disorders, smell

\section{Introduction}

The olfactory function is generated by chemical signals in the peripheral olfactory system and is important to human survival and perception. ${ }^{1}$ Olfaction has been extensively studied in the context of neurological diseases such as Parkinson's disease, ${ }^{1,2}$ Alzheimer's disease, ${ }^{3}$ multiple sclerosis (MS), ${ }^{4-9}$ and migraine. ${ }^{10}$ In patients with MS, olfactory dysfunction is associated with demyelination and the early axonal degeneration of olfactory brain regions, namely the inferior frontal and temporal lobes. ${ }^{4}$

Since the 1990s, several olfactory test protocols have been used. The most common ones are: the University of Pennsylvania Smell Identification Test, the Connecticut Chemosensory Clinical Research Center Test, and the Sniffin' Sticks Test. ${ }^{11}$ The Sniffin' Sticks Test is available for olfactory assessment in Turkey, Europe, and Australia. ${ }^{11}$ It is a well-designed olfactory test that the German Olfactology and Gustology Association ${ }^{12}$ recommends and uses in many studies that assess the olfactory functioning of MS patients.

The Sniffin' Sticks Test consists of a set of three olfactory tests that require the use of pen-like odor-dispensing devices. These three tests result in odor identification (OI), odor discrimination (OD), and odor threshold (OT) scores, which were recorded for this study. The sum of the three subtests is presented as a composite threshold discrimination identification (TDI) score. Normative data for olfaction using the Sniffin' 
Sticks test have been published in Turkey, but none of these studies include patients with MS. ${ }^{11}$

The present study assesses the olfactory impairment of patients with MS according to the duration of the disease, the patients' smoking habits, and their scores on the Mini-Mental State Examination (MMSE) and the Expanded Disability Status Scale (EDSS).

\section{Methods}

This study includes 30 healthy volunteers and 30 patients with MS (according to the McDonald Revised Criteria, 2005), ${ }^{13}$ who were evaluated in the Neurology Department of the Gazi University Faculty of Medicine. The study was carried out in accordance with the Declaration of Helsinki and approved by the Faculty of Medicine's ethics committee at Gazi University. All volunteers were at least 18 years of age and provided written informed consent prior to participating in this study. We recorded the participants' age, sex, and smoking habits. If participants had complaints about odor sensitivity, nasal surgery, head trauma, nasal polyps, rhinosinusitis, an upper respiratory tract infection, or about or any known olfactory diseases, then they were excluded from this study. The control group consisted of healthy individuals without neurological diseases or olfactory system abnormalities.

Clinical data were collected from MS patients and a neurological examination was administered. Patients who experienced an MS attack and who took steroids in the month prior to the study were also excluded. In addition, we also collected the following forms of data: the time since the patient's MS diagnosis, their history of optic neuritis, the medical therapy that they received for MS, as well as data from the EDSS and MMSE.

After providing demographic information and undergoing a clinical assessment, patients with MS and healthy controls performed the Sniffin' Sticks Test to evaluate their olfactory functioning. This test (Heinrich Burghart, GmbH, Wedel, Germany) ${ }^{14}$ includes three subtests that measure OT, OD, and OI. The scores of OT, OD, and OI were recorded. Finally the sum of the three test scores was calculated to provide the composite TDI score.

\section{Statistical analyses}

There was a $90 \%$ power of detecting true OT, OD, and OI score differences in the composite TDI scores when a minimum of 25 patients were included in each group, resulting in a $5 \%$ significance level. ${ }^{6}$ Statistical analyses were performed using the SPSS 15.0 software package (SPSS Inc., Chicago, IL, USA). Both $t$-tests and chi-squared tests were used to compare the demographic and clinical characteristics of the control and the MS groups. Within the MS group, a Pearson's correlation analysis assessed the relationship between the MS patients' clinical characteristics and their olfactory functioning. Twoway analysis of variance then examined the effects of smoking, headaches, and sex on each group. Values of $P<0.05$ were considered as statistically significant.

\section{Results}

\section{Demographic and clinical characteristics}

This study included 27 patients with relapsing remitting multiple sclerosis, three patients with secondary progressive multiple sclerosis, and 30 healthy controls. McDonald's Revised Criteria ${ }^{13}$ were used to diagnose MS. Demographic and clinical characteristics of the study participants are summarized in Table 1. The age of MS patients' ranged from 18 to 49 years (mean 34.3, standard deviation \pm 9.8 ), and the healthy controls were matched for age and smoking habits (Table 1).

\section{Olfactory functioning}

The results of olfactory tests were analyzed in terms of OT, OD, OI, and TDI scores. While examining the OD, OI, and

Table I Demographic and clinical characteristics of MS and control groups

\begin{tabular}{llll}
\hline Characteristics & MS & Control & $P$-value \\
\hline Age in years: mean, \pm SD (min-max) & $34.3, \pm 9.8(18-49)$ & $35.8, \pm 9.2(19-50)$ & 0.526 \\
Sex: $\mathrm{n}$ (\% female) & $21(70 \%)$ & $21(70 \%)$ & $0.24 I$ \\
Current smoking status: $\mathrm{n}$ (\% smokers) & $10(33.3 \%)$ & $9(30 \%)$ & 0.278 \\
MMSE: mean, \pm SD & $29.03, \pm 2.22$ & $29.97, \pm 0.18$ & $0.025 *$ \\
Disease duration in months: mean, \pm SD & $47.7, \pm 48$ & & \\
EDSS: mean, \pm SD & $1.91, \pm 1.57$ & & \\
Optic neuritis: $\mathrm{n}(\%)$ & $18(60 \%)$ & & \\
Immunomodulatory therapy: $\mathrm{n}(\%)$ & $25(83.3 \%)$ &
\end{tabular}

Note: $* p<0.05$.

Abbreviations: EDSS, Expanded Disability Status Scale; max, maximum; min, minimum; MMSE, Mini-Mental State Examination; MS, multiple sclerosis; SD, standard deviation. 
Table 2 Olfactory test scores for MS and control groups

\begin{tabular}{llll}
\hline Scores & MS & Control & $P$-value \\
\hline OT: mean, \pm SD & $11.04, \pm 3.28$ & $12.55, \pm 2.5 \mathrm{I}$ & $0.049 *$ \\
OD: mean, \pm SD & $10.83, \pm 2.30$ & $11.37, \pm 2.31$ & 0.375 \\
OI: mean, \pm SD & $11.60, \pm 2.50$ & $11.97, \pm 1.97$ & 0.531 \\
TDI: mean, \pm SD & $33.4, \pm 6.35$ & $35.89, \pm 5.48$ & 0.129 \\
\hline
\end{tabular}

Note: $* P<0.05$.

Abbreviations: MS, multiple sclerosis; OD, odor discrimination; OI, odor identification; OT, odor threshold; SD, standard deviation; TDI, threshold discrimination identification.

TDI scores, no differences between the MS and the control groups were found. However, the OT scores were higher in the control group than in the MS group $(P=0.49)$ (Table 2).

\section{Correlations between clinical characteristics and the results of olfactory tests}

An application of Pearson's correlation analysis revealed no correlation among demographic, clinical, and olfactory variables within the control group. Olfactory test scores of patients with MS were not associated with their MMSE scores, EDSS scores, disease duration, history of optic neuritis, or being on immunomodulatory therapy. However, the patients' ages were associated with their OI, OT, and TDI scores; older patients had lower OI, OT, TDI scores (Table 3).

In addition, the correlations between disease duration and the olfactory test results were specifically evaluated. In order to assess whether there were significant differences between the Sniffin' Sticks scores among these two groups, data from patients who had MS for $\geq 60$ months were compared to that from patients who had MS for $<60$ months. There was no significant difference.

\section{Discussion}

Previously, it was assumed that the odor function was spared in patients with MS. ${ }^{15}$ More recent investigations that rely on advanced techniques for odor testing have demonstrated impairment in relation to various aspects of olfaction in patients with MS. ${ }^{8}$ The Sniffin' Sticks Test is one of the most sophisticated ways of testing nasal chemosensory performance ${ }^{16}$ and previous reports using this test have established normative data for many countries, including Turkey. ${ }^{11,12,17,18}$ Tekeli et a $1^{17}$ provided an update of normative values for Sniffin' Sticks test in Turkish people and concluded that this test is applicable for clinical usage in Turkey without any cultural adaptation. The present study used the Sniffin' Sticks test to evaluate olfactory functioning in patients with MS, but these results did not demonstrate hyposmia in patients with MS. Compared with MS patients enrolled in other studies, $, 5,7$ this sample was relatively young and in the earlier stages of the disease. In general, the patients enrolled in the present study did not exhibit cognitive decline and cooperated fully with the examiner. Their EDSS scores were lower than those in previous studies, and this might have affected the results of the study. 5,7

Rolet et $\mathrm{al}^{7}$ observed that general damage to all three aspects of olfaction was found in $16 \%$ of patients. Primarily, these patients received natalizumab therapy and had more severe forms of MS when compared to the patients in this study. Furthermore, Silva et $\mathrm{al}^{5}$ found olfactory deficits in $68.8 \%$ of patients with secondary progressive multiple sclerosis but found no differences between those with relapsing remitting multiple sclerosis and a healthy control group. In this study, those in the later phases of MS did not demonstrate deterioration in their olfactory functioning. The patients who were diagnosed with MS at least 60 months prior to participating in this study had normal Sniffin' Sticks scores. This may be due to a low density of demyelinative lesions and relative absence of neurodegeneration.

Despite the fact that primary olfactory neurons are unmyelinated, the olfactory processing regions like inferior frontal and temporal lobes are myelinated. ${ }^{4,19}$ Magnetic resonance imaging studies investigating the influence of demyelinating lesions have shown a negative correlation between odor identification test scores and lesion load in olfactory regions of the brain. ${ }^{4,9}$ Doty et $\mathrm{al}^{4}$ proposed that a sufficient number of

Table 3 Correlations between age, MMSE and EDSS scores, disease duration, and olfactory test scores for patients with MS

\begin{tabular}{|c|c|c|c|c|c|c|c|c|}
\hline \multirow{2}{*}{$\begin{array}{l}\text { Olfactory } \\
\text { test scores }\end{array}$} & \multicolumn{2}{|l|}{ Age } & \multicolumn{2}{|c|}{ MMSE scores } & \multicolumn{2}{|c|}{ EDSS scores } & \multicolumn{2}{|c|}{ Disease duration } \\
\hline & $r$ & $P$-value & $r$ & $P$-value & $r$ & $P$-value & $\mathbf{r}$ & $P$-value \\
\hline OT & -0.365 & $0.047^{*}$ & 0.229 & 0.223 & -0.286 & 0.125 & -0.127 & 0.505 \\
\hline OD & -0.237 & 0.207 & 0.385 & 0.036 & -0.142 & 0.454 & -0.261 & 0.164 \\
\hline Ol & -0.412 & $0.024 *$ & 0.450 & $0.013^{*}$ & -0.321 & 0.084 & -0.161 & 0.395 \\
\hline TDI & -0.436 & $0.016 *$ & 0.435 & $0.016 *$ & -0.325 & 0.079 & -0.223 & 0.235 \\
\hline
\end{tabular}

Note: $* P<0.05$.

Abbreviations: EDSS, Expanded Disability Status Scale; MMSE, Mini-Mental State Examination; MS, multiple sclerosis; OD, odor discrimination; OI, odor identification; OT, odor threshold; TDI, threshold discrimination identification. 
plaques within olfactory eloquent regions of the brain give rise to olfactory dysfunction.

Lutterotti et $\mathrm{al}^{6}$ identified impaired OT and OI test results in MS patients with Sniffin' Sticks Test. They found that the impairment of patients' odor thresholds was more prominent in the early phases of MS, and they assumed that this was a result of clinical disease activity related to inflammation. Longer disease durations affected odor identification. Moreover, Rolet et $\mathrm{al}^{7}$ observed that $40 \%$ of patients with MS had odor thresholds that were the primarily affected aspect of olfactory function.

We can speculate that the progression of the disease and its acute inflammatory process influence the olfactory dysfunction. However, this study did not find any difference between patients with a disease duration of $<60$ months and those with disease duration of $\geq 60$ months, indicating that olfaction is generally spared prior to the onset of neurodegeneration. This may be a result of the fact that the impairment in OT scores of MS patients is associated with the duration and the course of the disease. As patients with acute attacks in the previous month were excluded, this study could not determine whether the inflammatory phase initiated damage in the olfactory regions.

Olfactory loss is considered as a major consequence of aging and may be the early sign of neurodegenerative diseases. This could be the result of changes in olfactory bulb, degeneration of primary and secondary olfactory cortices. ${ }^{20}$ Olfactory dysfunction's prevalence in dementia may be as high as $100 \%$ in Alzheimer's disease, $90 \%$ in Parkinson disease, $96 \%$ in the frontal variant of frontotemporal dementia, and $15 \%$ in vascular dementia. ${ }^{21}$ MS-related olfactory loss is related to the number of plaques in olfactory regions (inferior frontal and temporal lobes $)^{8}$ and may be a marker of disease progression. Zivadinov et $\mathrm{al}^{8}$ stated significant correlation between olfactory dysfunction and symptoms of anxiety, depression, and severity of neurological impairment.

\section{Limitations}

There are some limitations to our study. First, the group of participants was relatively small. Second, the study included mostly patients with relapsing remitting multiple sclerosis. The progressive courses of MS would lead to different levels of olfactory dysfunction. Third, EDSS scores were relatively low showing that the patients were mildly affected with MS. Fourth, the patients with acute attacks were excluded; hence, the effects of acute inflammatory process on olfactory function remained unknown. Fifth, magnetic resonance imaging data of patients were not included in the study. The presence of MS plaques in olfactory regions would have been enlightening. Further studies are needed to assess the correlation between plaque burden and olfactory dysfunction in MS. Sixth, the neurocognitive tests were not applied elaborately, only the MMSE was completed. The effects of cognitive deficits on olfactory functioning were not analyzed.

\section{Conclusion}

In recent studies, odor threshold impairment seemed to be the most striking finding in patients with MS. Although the present study found a mild alteration in OT, olfactory dysfunction appears to be a consequence of neurodegeneration in the higher-order olfactory brain regions, which is thought to be a time-dependent process.

\section{Acknowledgments}

This research was presented at the World Congress of Neurology, October 31 to November 5, 2015, in Santiago, Chile. The abstract has also been published in a supplement of the Journal of Neurological Sciences.

\section{Disclosure}

The authors report no conflicts of interest in this work.

\section{References}

1. Haehner A, Boesveldt S, Berendse HW, et al. Prevalence of smell loss in Parkinson's disease - a multicenter study. Parkinsonism Relat Disord. 2009;15(7):490-494.

2. Doty RL. Olfactory dysfunction in Parkinson disease. Nat Rev Neurol. 2012;8(6):329-339.

3. Forster S, Vaitl A, Teipel SJ, et al. Functional representation of olfactory impairment in early Alzheimer's disease. J Alzheimers Dis. 2010; 22(2):581-591.

4. Doty RL, Li C, Mannon LJ, Yousem DM. Olfactory dysfunction in multiple sclerosis. Relation to plaque load in inferior frontal and temporal lobes. Ann N Y Acad Sci. 1998;855:781-786.

5. Silva AM, Santos E, Moreira I, et al. Olfactory dysfunction in multiple sclerosis: association with secondary progression. Mult Scler. 2012; 18(5):616-621.

6. Lutterotti A, Vedovello M, Reindl M, et al. Olfactory threshold is impaired in early, active multiple sclerosis. Multiple Scler. 2011;17(8):964-969.

7. Rolet A, Magnin E, Millot JL, et al. Olfactory dysfunction in multiple sclerosis: evidence of a decrease in different aspects of olfactory function. Eur Neurol. 2012;69(3):166-170.

8. Zivadinov R, Zorzon M, Monti Bragadin L, Pagliaro G, Cazzato G. Olfactory loss in multiple sclerosis. J Neurol Sci. 1999;168(2):127-130.

9. Zorzon M, Ukmar M, Bragadin LM, et al. Olfactory dysfunction and extent of white matter abnormalities in multiple sclerosis: a clinical and MR study. Mult Scler. 2000;6(6):386-390.

10. Saisu A, Tatsumoto M, Hoshiyama E, Aiba S, Hirata K. Evaluation of olfaction in patients with migraine using an odour stick identification test. Cephalalgia. 2011;31(9):1023-1028.

11. Orhan KS, Karabulut B, Keles N, Deger K. Evaluation of factors concerning the olfaction using the Sniffin' sticks test. Otolaryngol Head Neck Surg. 2012;146(2):240-246.

12. Hummel T, Kobal G, Gudziol H, Mackay-Sim A. Normative data for the "Sniffin' Sticks" including tests of odor identification, odor discrimination, and olfactory thresholds: an upgrade based on a group of more than 3,000 subjects. Eur Arch Otorhinolaryngol. 2007;264(3):237-243. 
13. Polman CH, Reingold SC, Edan G, et al. Diagnostic criteria for multiple sclerosis: 2005 revisions to the "McDonald Criteria". Ann Neurol. 2005;58(6):840-846.

14. MediSense. Burghart Sniffin' Sticks - Burghart Smelltests. Available from: http://smelltest.eu/en/burghart-sniffin-sticks-burghart-smelltests/? gclid=CN263sCi2s0CFZSEaQodyrsOvQ. Accessed August 11, 2016.

15. Ansari KA. Olfaction in multiple sclerosis. With a note on the discrepancy between optic and olfactory involvement. Eur Neurol. 1976; 14(2):138-145.

16. Hummel T, Sekinger B, Wolf SR, Pauli E, Kobal G. 'Sniffin' sticks': olfactory performance assessed by the combined testing of odor identification, odor discrimination and olfactory threshold. Chem Senses. 1997;22(1):39-52.

17. Tekeli H, Altundağ A, Salihoğlu M, Cayönü M, Kendirli MT. The applicability of the "Sniffin' Sticks" olfactory test in a Turkish population. Med Sci Monit. 2013;19:1221-1226.
18. Oniz A, Erdogan I, Ikiz AO, Evirgen N, Ozgoren M. The modified Sniffin' sticks test in Turkish population based on odor familiarity survey. J Neurol Sci. 2013;30:270-280.

19. Braak E, Braak H, Strenge H. The fine structure of myelinated nerve cell bodies in the bulbus olfactorius of man. Cell Tissue Res. 1977;182(2): 221-233.

20. Barresi M, Ciurleo R, Giacoppo S, et al. Evaluation of olfactory dysfunction in neurodegenerative diseases. J Neurol Sci. 2012;323(1-2): 16-24.

21. Alves J, Petrosyan A, Magalhaes R. Olfactory dysfunction in dementia. World J Clin Cases. 2014;2(11):661-667.

\section{Publish your work in this journal}

Neuropsychiatric Disease and Treatment is an international, peerreviewed journal of clinical therapeutics and pharmacology focusing on concise rapid reporting of clinical or pre-clinical studies on a range of neuropsychiatric and neurological disorders. This journal is indexed on PubMed Central, the 'PsycINFO' database and CAS, and is the official journal of The International Neuropsychiatric Association (INA). The manuscript management system is completely online and includes a very quick and fair peer-review system, which is all easy to use. Visit http://www.dovepress.com/testimonials.php to read real quotes from published authors.

Submit your manuscript here: http://www.dovepress.com/neuropsychiatric-disease-and-treatment-journal 